\title{
A Conversation with Donald Peterson
}

$\mathbf{T}$ hat light we see at the end of the tunnel is the convergence of voice and data communications with business applications. As chairman and chief executive officer of Avaya, Donald Peterson is in a position to help make that convergence happen sooner rather than later.

Peterson has been with Avaya since it was spun off from Lucent in 2000. Prior to that he was chief financial officer of AT\&T's Communication Services Group and Lucent.

Peterson began his career in 1973 as a senior analyst at State Mutual Life Assurance Company. He joined Nortel Networks in 1976 and eventually was appointed president of Nortel Communications Systems before joining AT\&T in 1995. Peterson attended Worcester Polytechnic Insti-

\section{What will the}

COMING REVOLUTION

MERGING VOICE AND

DATA COMMUNICATIONS

WITH BUSINESS

APPLICATIONS BRING? present, and future of telecommunications is Lucy Sanders, formerly an R\&D VP and chief technology officer in Avaya's CRM business. Now retired from those positions, Sanders still watches the industry's progress from a frontrow seat. She now serves as executive in residence at the University of Colorado's ATLAS (Alliance for Technology, Learning and Society) Institute, whose mission includes bridging information technology with the arts, humani-

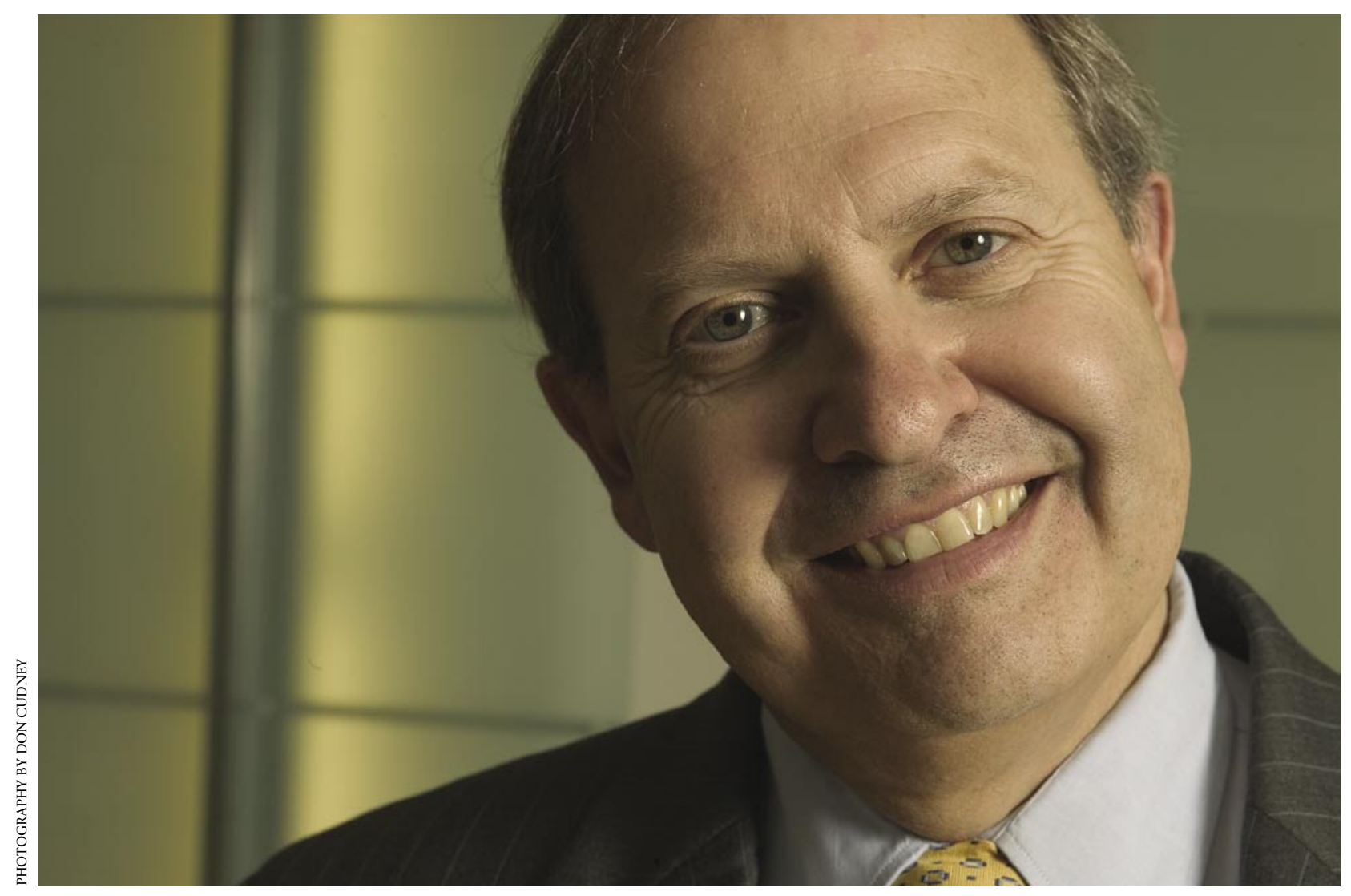


ties, and social sciences. She is also CEO of the National Center for Women and Information Technology and serves on a number of nonprofit and high-tech company boards.

Sanders received the Distinguished Engineering Alumni Award from the College of Engineering at the University of Colorado at Boulder and is a recipient of the Bell Labs Fellow Award. She holds five patents in telecommunications

LUCY SANDERS Tell us a little bit about your experience with Avaya.

DONALD PETERSON When Lucent decided to spin off Avaya, I raised my hand and said I'd really like to be with that side of the business. I like the enterprise customers; I like the more rapid adaptation and utilization of technology. You have to be careful when you raise your hand. Sometimes you get what you ask for.
The reality of that didn't come to light until 1999 to 2000. Then we were confronted with the next step of implementation. As soon as voice got on the IP networks, the technologies that are used to make IP networks work well-like buffering, re-sending lost bits, and so forthwere recognized as in fact detrimental to good voice communications. So we had to understand what the network would have to look like now to accommodate voice communications.

In the last 18 months, I think we've figured out how to make voice really work in an IP environment. We've been waiting for that, and now we're ready to take off.

LS Can you speak a little bit about the killer apps for VoIP?

DP Everybody wants a killer app, particularly investors, because they want to get in on the landslide. At Avaya we

\section{In the long term, we're dealing with a truly transformational technology for businesses.}

Avaya has been a great experience. The first year was actually pretty good. The next two were real challenges. In many ways our fortunes bottomed out at the end of 2002. We had a really nice rebuilding year in 2003, and we're continuing that in 2004 . So we're not through all of the challenges, but we're definitely on a good trend with product and customers.

LS You can't pick up any magazine or newspaper without reading about voice over IP (VoIP). Why now, and not 10 years ago? Why now, and not five years in the future? It just seems like it's everywhere.

DP I think the technology to do voice-over packets at the base level has been around 10 years. Frankly, the quality-of-service benefits of some of the competing protocols, such as frame relay, looked for a while like a better solution. But the reality of IP as the winner for data and the reality that a convergence of networks to a single technology was the optimal solution drove the industry to a selection of IP as the right next technology for voice. distinguish VoIP from IP telephony. Voice over IP is the packetized carriage of voice and the transport. That's been done for a long time actually, and in my view it's very reliable today and it's going to keep growing.

IP telephony is the application of voice within that environment, and that's in the very early stages of understanding what the real opportunity is. In the long term, we're dealing with a truly transformational technology for businesses. Right now, the early applications that have caught on are contact centers. That has been a particularly important application because there is so much networking value in the kinds of costs in a contact center. Those have been early adopters. The extreme manifestation is the location of agent groups outside of North America, even though they serve North American markets. That's obviously a political thing, but leaving that aside, that has been a killer app and has driven a lot of the earliest utilizations of IP telephony.

As we go forward, IP telephony and its follow-on technologies, such as SIP (session initiation protocol), are going to enable a knowledge in the network that can in turn drive applications and then communication 


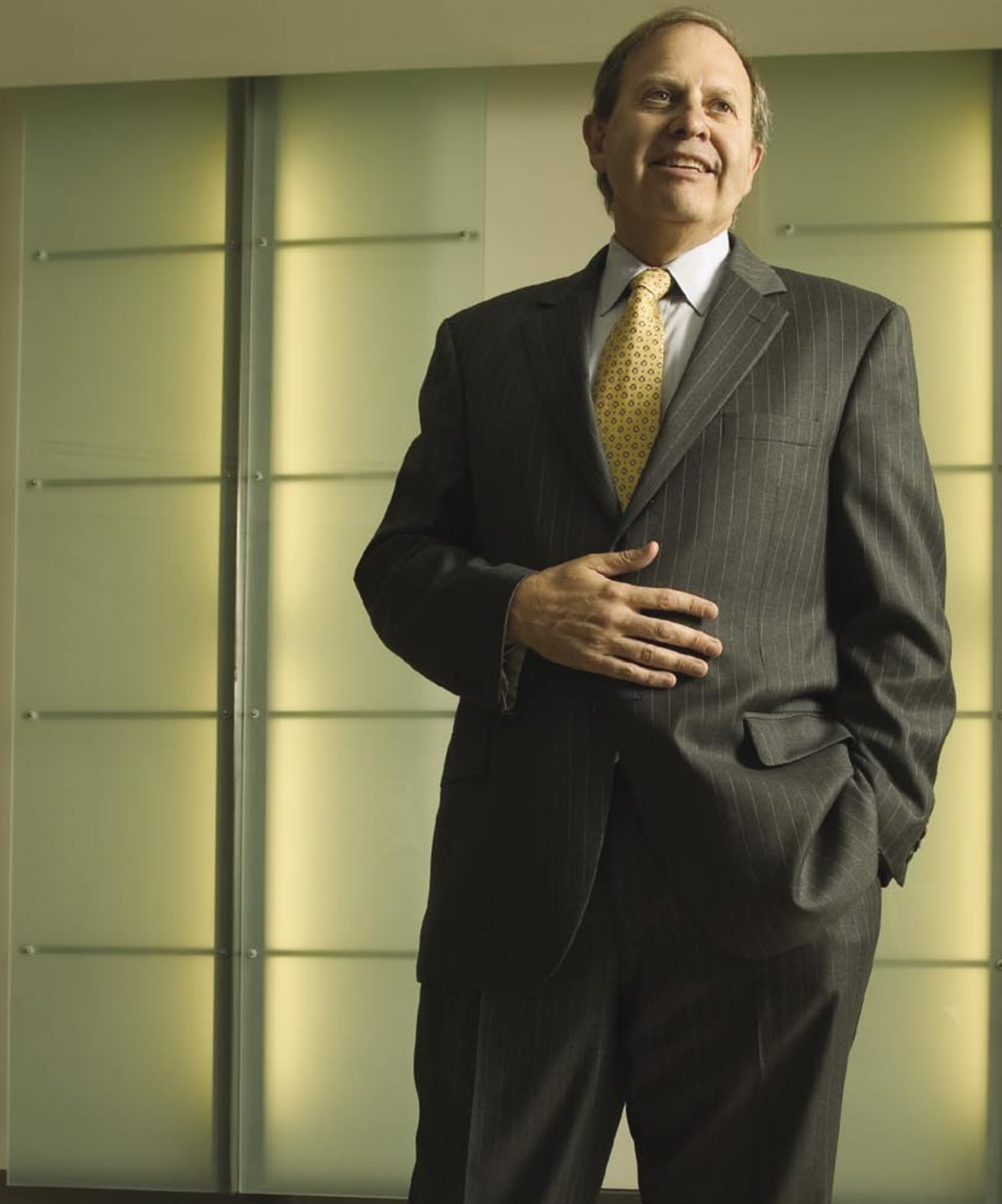




\section{interview}

capabilities that will be reminiscent of 2001 and Hal the computer-but without any of the malevolence, I hope, that was in that movie.

We've gone through a stage where the network has enabled an any-to-any connection-anybody, anywhere, anytime, anyplace. That's been done. Today, we are at a stage of the right person at the right time in the right place. Whereas we used to call a location, we now can call a device. In the future, we're going to call a person and it's going to be the right person because the application is going to know who the right person is, and the network is going to know the person's availability. Those things are going to get married, and the right thing is going to happen at the right time in a very optimized way.

Adaptation and actual usage is a number of years in the future. But the path is very clear going forward. Think about whole companies becoming call centers-any employee is available to any customer at any time with the information needed to really be of use to that customer. That can be done. It's just a matter of optimizing it so that there's value in doing it-value meaning benefit in excess of cost.

LS You're hitting on one of my favorite application areas: the ability to pick my favorite call-center agent, perhaps who's worked with me before, who happens to be available when I want to call, because I know he or she is available.

DP If you have a customer with a favorite agent, as a company you must make a decision: do you make the preferred agent available or do you make available a bet-

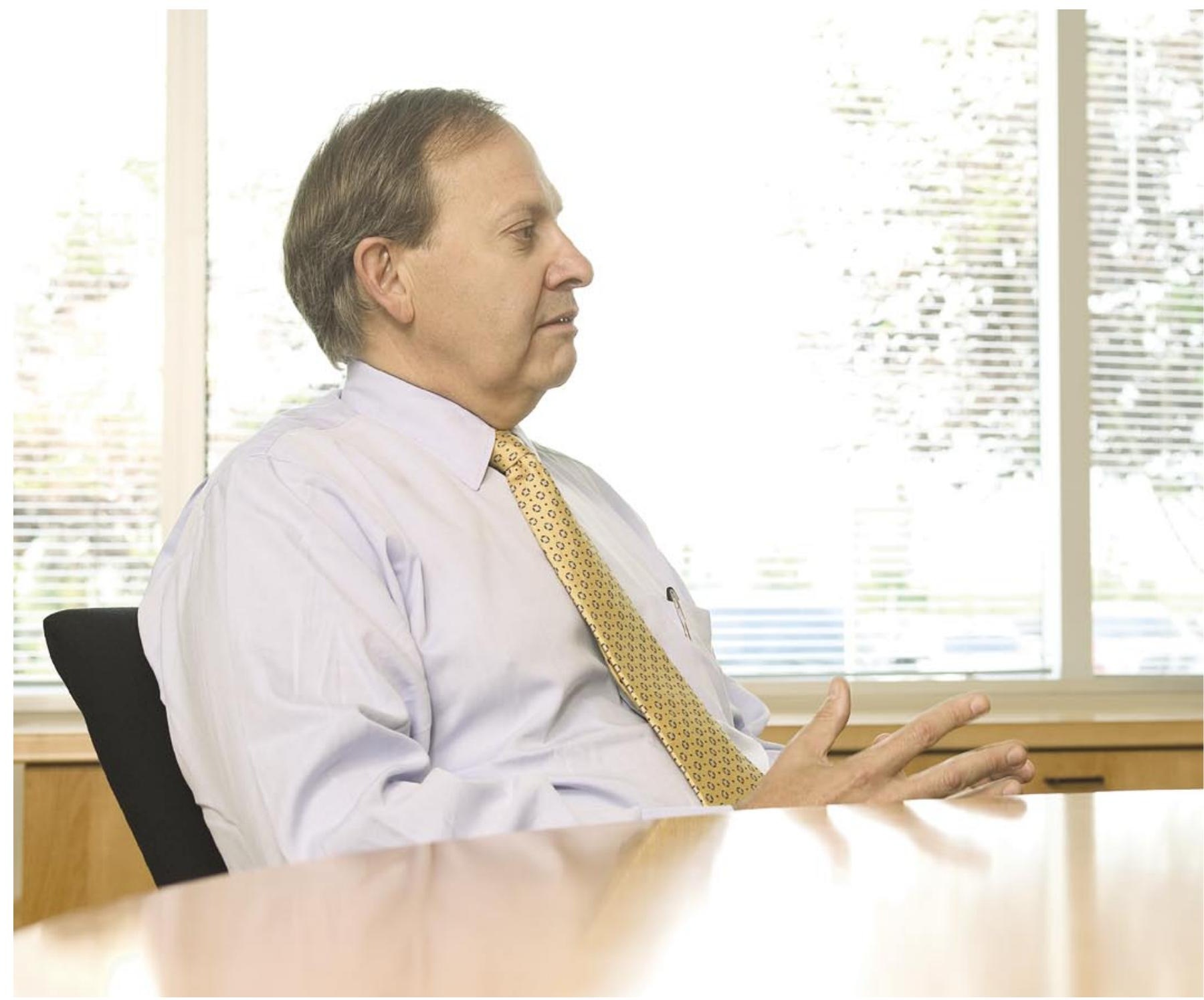


ter agent who could be more successful selling. There's not a generic answer to that. If that customer is calling with a complaint, I would opt for the favorite agent. If you're calling that customer with a new product, I might opt for a more capable agent-who could be a friend of the first agent and get into the loop on an emotional basis, yet could deliver a quality of service that perhaps the first agent couldn't.

There's no reason why we can't have the right person at the right time with these new technologies. That's what the integration of communications with applications is all about.

LS Then when you blend that into the different media streams-for example, video-do you see any other applications? Having things over the same network certainly enables you to have a convergence of the different media.

DP We're going to see convergence, for sure. But at a logical level, we're going to see choices being made on the optimal way to communicate, which is going to be not only a function of what you're communicating from where, but also a function of who you're communicating with and where that person is and what his or her capability is.

For instance, we're doing a lot of work on collaboration and conferencing. We're recognizing that some people who should be in any particular conference may have different technical capabilities at the moment than others. So if there are six people who should be in this trading opportunity at an investment bank, the traders on the desk might have three or four screens in front of them and you want to give them a lot of information and enable them to com- municate easily with voice and e-mail and over the Web and so forth.

But the decision-maker may in fact be in a car and have only a cellphone with a two-by-two-inch screen. That person still may want to be aware of much of what the trader sees. So we'll be looking for the network in essence to arbitrate the information, recognize the capabilities of the devices that each user has, change the display process of the information in a way that what goes there is the essence of what the user needs, and then put that person in the conference.

If it's only with a RIM (BlackBerry) device, so be it. Let that person work his thumbs as hard as he can and stay in the conference. If somebody else is on a phone, do the voice-to-text translation so that the person with the RIM can see what's going on in the voice conversation as well. That's one of the offers that I believe the network is going to make in this converged world, and I think it's going to be a highly valued one, once we're all used to it.

LS What kinds of regulatory issues do you see around VoIP? Do the regulatory issues impact enterprise businesses in the same way they impact the consumer space-for example, 911 [emergency call systems]? DP My view is that our regulatory regimes are out of sync with the real world. We have politics-not in a malevolent sense, but in a small $p$ sense-in charge of regulating a significant part of this solution set: telephone companies, cable companies, and so forth. And we have a political decision, perhaps with a capital $P$, that says we don't want to regulate the IP part of this and the Web part of this.

These are all part of the same whole. We run the risk of eliminating some of the things we take for granted in the old world as we go out of our way to enable the new world with competitive help.

The Federal Communication Commission's wireless enhanced 911 (E911) rules [providing 911 dispatchers with additional information on wireless 911 calls] do not apply in the same way when you get into Web-based telephony. A really glaring example is CALEA (Communications Assistance for Law Enforcement Act), requiring wireless and wireline companies to enable law enforcement agencies to access conversations in the wide area. LS That's one of my favorites.

DP It's wiretapping, right? We've made a decision as a country that we're going to enable wiretapping. We forced wireline carriers to provide capabilities to do it. IP telephony, we're saying, is not subject to the same thing. 
We are really fooling ourselves if we believe that the bad guys are going to be anywhere but over on the IP telephony side. Why would they use the old stuff, knowing that it's exposed in this way, if the other stuff were available and cheaper and more secure for their purposes?

The regulatory regimes have to realize that they can't tax and tax the old and free up the new, call it competition, and think it's going to last. There's a huge crosssubsidy in terms of infrastructure that moves from the wireline side to the Web side. If they bankrupt the wireline side, the Web goes, too. The politicians are going to have to deal with the inconsistencies of the regulation and the inconsistencies of their position-or the market will take it away, anyway. But I see very little in the way of a political appetite to grapple with this issue, unfortunately.

LS Should businesses that use VoIP have any extra security concerns about their voice network being hacked?

DP Today they should. The VoIP network is probably the most porous thing we've had since you could walk into your neighbor's yard with your wireless phone and listen to your neighbor's conversation. We did a demonstration at the VoiceCon meeting a few months ago. I was giving a talk and I had one of our technicians dial into an ISP server that served his home and make a phone call to his house. He played back his conversation on the phone from the ISP server with a short delay. All he had going for him was a PC on the stage of the convention hall.

This stuff is in many, many cases all in the clear, and people are wholly unaware that it is. At Avaya, all of our conversations are encrypted; all of our call control is encrypted as well. So that couldn't happen in an Avaya environment. But for the consumer today, you should assume that IP telephony is in the clear until you know differently.

LS I have talked to some people who believe a lot of this [lack of security] is because of the different vendors involved and the different points of interoperability-and not enough agreement about what needs to get done. DP And part of it is the proliferation of service providers, many of whom are unregulated because they have this virtuous position of using IP technology. I can't understand why that virtue supersedes all others, like E911, like security and privacy in your conversation, and so forth. Twenty-five years ago if you recorded or listened to somebody's call, you'd go to jail. Wiretapping was a federal crime. Today, it may be a federal crime but nobody is looking anymore, and, in fact, it has been enabled by the shift to this new technology.
LS Let's put ourselves out in the future just a little bit. Ten years from now, what will VoIP be like?

DP I'm going to go out on a limb and say VoIP is an access technology. I think the networks will be using different protocols unless IP evolves and becomes something else (and we just call it IP because it fits the marketing model). But there's going to be fiber-there is fiber in all interoffice communications. None of that is IP managed. Much of the office-to-office stuff is frame relay. There's no advantage to moving that to IP. You get better quality-ofservice control today using frame relay.

The office-to-office network is going to continue to drive down that road. Probably in 10 years it will be all fiber. Having said that, the access loop 10 years from now is going to be a combination of TDM (time division multiplexing) and some wireless and IP telephony. I think 10 years out, probably one-half to two-thirds of the base will be IP telephony, but there will still be a very large component that is essentially TDM.

We will see enterprises looking for applications that optimize the value of the applications to all the end users, including TDM endpoints. So CIOs and others are going to have to worry about a mixed technology network for a long, long time. But we're clearly on a path to migrate to IP telephony in enterprises, as well as in the access loop for consumers. I believe that's the right path.

I think we have to provide for these important valueadded attributes-E911, security, CALEA, and so forth. But the underlying technology path is clear. We'll be just at the beginning 10 years from now, I think, of really realizing the value of communication applications with business applications and robustly taking this knowledge of presence and capability that's in the network realtime and merging it with the value that's in the business application of selling insurance, or underwriting in an accident situation, or trading on your stock account, or whatever it is.

We have yet to define how those will be married together and what the attributes of that sharing will be. Of course, the communications companies won't define it, the users will. We'll be part of the enablement of it, and I think we'll be robustly down that path in 10 years, but only starting to see the business transformational impacts that this combination is going to have in the future.

LS I think our readers want to know if we're going to have a Star Trek scenario-you know, a "Beam me up, Scotty" kind of thing?

DP The technology is there. You can wear a device on your belt, use a Bluetooth connection to an earpiece, 
have a microphone embedded under the crown in your mouth, have a hologram coming out of your eyeglasses so you can have essentially a screen in front of you. By the way, that was a description in a book called What Will Be, by Michael Dertouzos (Harper San Francisco, 1998), who ran the MIT Laboratory for Computer Science.

But most of us will still want a device with an on/off button so we can shut it down.

LS In your position, you obviously go around and talk about voice over IP to lots of enterprise executives. What is the most frequently asked question that you get when you're out talking about the technology?

DP My answer is to a slightly different question: What is the hardest question that people ask? And that is, "How do you get from here to there?" If you were building it all new, you would know exactly what to do. But how to integrate it when you've got an established business that has telecommunicationsand you don't have all the money in the world just to throw out the old and bring in the new-is an extremely complicated question.

You can obviously see how to migrate node by node. But when you do that, you really want to retain the value of your applications across all of the users and across all the applications. So that incrementality to the migration plan is really the most complex question. There are not a large number of people with the skills to take these applications and these communication capabilities and optimize them on both technologies at the same time. Those are the people we have to grow very, very rapidly in order for this to work, because almost nobody can afford what we used to call in the PBX business, "the forklift upgrade."

You could take one box out and replace it, but you can't take your whole network out and replace it. There is a better way. That's what we work with our customers on, but it's always situational-specific and customer-specific-and it's always complicated. That's the toughest thing we're going to have to deal with.

LS Any parting shots?

DP I think it's important to keep in mind-notwithstanding the flexibility and complexity of it - the inevitability of it. My advice to people would be to create the vision of where you want to be in that five- to 10year period and then make sure your investments at least have a part of the vector in that path.

That way, over time you will get there. And at some point, you can pull all the rest out and just make it all happen. There was that point in analogto-digital conversions, and it had a huge payoff, particularly with carriers in the central office environment. Once they had 75 percent of their offices on digital, taking that last 25 percent out from analog to digital was dramatic in terms of its effect on managing the network, training people, keeping spare parts. But in order to do that, you have to make sure that each of these incremental steps takes you toward your goal.

LS Very much an evolution.

DP An old professor of mine used to call it logical incrementalism. I think that captures it. Know where you want to go and logically make sure you're always taking a step in that direction, even if you have to veer off and wrap in some other value propositions as you do it. You'll get there in the end. $Q$

\section{LOVE IT, HATE IT? LET US KNOW}

feedback@acmqueue.com or www.acmqueue.com/forums 